\title{
FISHji: New ImageJ macros for the quantification of fluorescence in epifluorescence images
}

\author{
Sílvia Fontenete ${ }^{\mathrm{a}, \mathrm{b}, \mathrm{c}, \mathrm{d}, \mathrm{e}, 1}$, Daniel Carvalho ${ }^{\mathrm{a}}$, Anália Lourenço ${ }^{\mathrm{f}, \mathrm{g}}$, Nuno Guimarães ${ }^{\mathrm{a}, \mathrm{b}, \mathrm{c}, \mathrm{d}}$, \\ Pedro Madureira ${ }^{b, e, h}$, Céu Figueiredo ${ }^{b, c, i}$, Nuno Filipe Azevedo ${ }^{a, *}$ \\ a LEPABE, Laboratory for Process Engineering, Environment, Biotechnology and Energy, Faculty of Engineering, University of Porto, Porto, Portugal \\ b $i 3 S$, Instituto de Investigação e Inovação em Saúde, Universidade do Porto, Porto, Portugal \\ ' IPATIMUP, Instituto of Molecular Pathology and Immunology, University of Porto, Porto, Portugal \\ d Nucleic Acid Center, Department of Physics, Chemistry and Pharmacy, University of Southern Denmark, Odense M, Denmark \\ e ICBAS, Institute of Biomedical Sciences Abel Salazar, University of Porto, Porto, Portugal \\ ${ }^{\mathrm{f}}$ ESEI-Escuela Superior de Ingeniería Informática, Universidad de Vigo, Ourense, Spain \\ g Centre of Biological Engineering, University of Minho, Braga, Portugal \\ h IBMC, Institute for Molecular Biology and Cell Biology, Porto, Portugal \\ ${ }^{\mathrm{i}}$ FMUP, Faculty of Medicine of the University of Porto, Porto, Portugal
}

\section{A R T I C L E I N F O}

Article history:

Received 5 November 2015

Received in revised form 27 January 2016

Accepted 4 April 2016

Available online 9 April 2016

\section{Keywords:}

Biomedical

RNA

LNA

Image analysis

Optimisation

Computational image processing

\begin{abstract}
A B S T R A C T
Fluorescence in situ hybridization (FISH) is based on the use of fluorescent staining dyes, however, the signal intensity of the images obtained by microscopy is seldom quantified with accuracy by the researcher. The development of innovative digital image processing programs and tools has been trying to overcome this problem, however, the determination of fluorescent intensity in microscopy images still has issues due to the lack of precision in the results and the complexity of existing software.

This work presents FISHji, a set of new ImageJ methods for automated quantification of fluorescence in images obtained by epifluorescence microscopy. To validate the methods, results obtained by FISHji were compared with results obtained by flow cytometry. The mean correlation between FISHji and flow cytometry was high and significant, showing that the imaging methods are able to accurately assess the signal intensity of fluorescence images. FISHji are available for non-commercial use at http://paginas.fe. up.pt/ nazevedo/.
\end{abstract}

(c) 2016 Elsevier B.V. All rights reserved.

\section{Introduction}

In science, digital images are a crucial piece of information. The ability to process and analyse the large volume of images produced by the plethora of microscopy techniques available raises the need for specialized software tools [1]. Images should be processed in a systematic and standardized way, such that results are comprehensible and reproducible. There are a number of commercial and open source image processing tools available, and the range of imple-

Abbreviations: FISH, fluorescence in situ hybridization; LNA, locked nucleic acid; LoG, Laplacian of Gaussian; MFI, mean fluorescence intensity; PI, propidium iodide; ROIs, regions of interest; TSA, trypticase soy agar; $2^{\prime} O M e, 2^{\prime}-O$-methly RNA.

* Corresponding author.

E-mail address: nazevedo@fe.up.pt (N.F. Azevedo).

1 New address: Epithelial Cell Biology Group, Cancer Cell Biology Program, Spanish National Cancer Research Centre, Madrid, Spain. mented functionalities is significant [2-10]. However, the general purpose of most of these tools hampers domain or applicationspecific use by laymen, i.e. it is not straightforward for a researcher to use such general software to analyse biological images, without understanding the specifics of the image processing and analysis algorithms. As such, the development of end-user biological image analysis tools is considered useful, essentially for analyzing fluorescence results obtained by diagnostic methodologies for microorganisms. These methodologies or techniques use staining with fluorescent binding dyes which are then visualized using fluorescence microscopy. In order to have a quantitative analysis of the results, the fluorescence intensity needs to be accurately measured.

Fluorescence in situ hybridization (FISH) is one of the methodologies in which a quantitative validation of results is required. FISH has been used as a molecular tool for the analysis and detection of microorganisms [11]. Since the first application in 1989 by Delong et al. [12], this method has undergone several improvements in 
order to overcome limitations, such as low fluorescence intensity $[13,14]$. This evolution of the method has led to the use of new types of nucleic acids such as locked nucleic acid (LNA) and 2'-O-methly RNA ( $\left.2^{\prime} \mathrm{OMe}\right)[14,15]$. Other chemical modifications within nucleobases, ribose or deoxyribose, and within phosphate moieties have been also introduced to improve the resistance to nucleases present in the cells or body fluids [16-18]. In FISH experiments, the evaluation of the results is based on microscopy, which the quantitative assessment is performed by the researcher, which is dependent of the observer interpretation [19], or on flow cytometry, that provides an automated quantitative assessment but requires the use of expensive equipment and is only applicable for cells in suspension. Therefore, computerized image processing tools offer the most promising and versatile approach to minimize costs and overcome the variability of human microscope analysis. Daime et al. developed a digital image analysis program (DAIME) especially used for microbial ecology [20]. Although, DAIME has different features such as analyzing 2D and 3D microscopy datasets of microorganisms stained by FISH, this program is complex, is generally used for environmental bacteria [21-23] and no validation studies against flow cytometry have been made so for. ImageJ is another well-known and publicly available image processing tool (http://rsbweb.nih. gov/ij/). It has released many plugins and macros useful to biomedical image processing [24], however, also in this case there are no comparative studies against flow cytometry to confirm the use of this software in fluorescence quantification of microscopic images.

This article describes five different methods for the quantification of fluorescent intensity in microscopic images (named FISHji). As a case study, LNA probes were applied in different hybridization conditions, and evaluated by microscopy and cytometry approaches. The biological goal was the detection of 16S rRNA in the clinically relevant bacterium Helicobacter pylori. The developed methods are freely available and integrated in the Image J software package.

\section{Experimental procedures}

\subsection{Workflow of FISHji methods and validation}

In this work, three semi-automatic and two automatic FISHji methods were created (Fig. 1). To validate these methods, the FISH procedure was performed both in bacterial suspensions and in attached bacteria. Eighteen LNA probes specifically designed for $H$. pylori were used at different temperatures, in order to obtain a large range of fluorescence intensities. All images obtained by microscopy (attached bacteria) were analysed by the five FISHji methods and these results were compared with cytometry data (bacterial suspensions) hybridized under the same conditions (temperature and buffer). Afterwards, all results were statistically analysed and the correlation between each FISHji method and flow cytometry was performed. Finally, an extra validation was then performed using another fluorescence staining procedure (propidium iodide) for the FISHji methods.

\subsection{Fluorescence analysis and FISHji architecture}

In terms of operational mode, there are three semi-automatic (FISHji1, FISHji2 and FISHji3) and two automatic approaches (FISHji4, and FISHji5) (Fig. 2). For all methods, the channels of the original RGB (red, green, and blue light) images were separated in order to analyse the channel where fluorescence is emitted.

The methods consist of three sequential steps: optimization, segmentation and analysis, and measurement steps. In the optimization module, pixel-based treatments are performed in order to highlight the regions of interest (ROIs) and allow the removal of artefacts. In the segmentation module a default black and white threshold supports cell segmentation. The ROIs are outlined and artefacts are discarded by the command "Analyse Particles". The mean fluorescence intensity (MFI) is then calculated as the average of each ROI (Fig. 2).

For FISHji1, optimization treatments are not applied. In the FISHji2 and FISHji3, a background subtraction based on the "rolling ball" algorithm (radius $=80$ pixels) is performed to the green channel in order to reduce background heterogeneity and the presence of artefacts [25]. In the FISHji2 method, the obtained image is segmented and further analysed to determine the MFI of the image. In FISHji3, the brightness and contrast features of the corrected image are manually adjusted using the "B/C Adjustment" (Brightness and Contrast) tool to further enhance the foreground. Subsequently, the adjusted image is thresholded and a binary mask is created. The ROIs present in the mask are redirected to the corrected image and MFI is quantified by the ROI manager interface.

In the automatic FISHji4 and FISHji5 methods, the corrected image is convoluted using a Laplacian of Gaussian (LoG) filter $(9 \times 9$ kernel) [26]. In the FISHji5 method, an automatic B/C adjustment of the corrected image is performed before the convolution. First, a $\mathrm{B} / \mathrm{C}$ adjustment based on the image's histogram is automatically applied and then the convolution step was performed. In addition, the binary operation "Fill Holes" is applied to fill intra-aggregate spaces and maintain ROI integrity, providing an optimal binary mask.These optimization steps (except "Rolling Ball") change the real pixel values of the image, including the ROIs. So, a duplicated image is taken from the corrected image in order to allow enhancement of foreground pixels without compromising MFI quantification. After segmentation, the ROIs defined by the binary mask are redirected to the duplicated image, and only then the MFI is calculated. Thus, the MFI is determined from the real pixel values of the Corrected Image, outlined by the binary mask overlay.

All FISHji methods were implemented as macros in Image (version $1.49 \mathrm{o}$ or higher). The analysis parameters were set as 100-600 pixels for the minimum and maximum valid object size (i.e. pixel $\hat{2}$ ) and $0.1-0.7$ for the range of circularity i.e. $4 \times \mathrm{pi} \times($ area/perimeter $\hat{2})$. These values were established by experimental trials in $\mathrm{H}$. pylori, and taking into account the real size and shape of the bacterium. Images were not calibrated since all had the same resolution $(1392 \times 1040 \mathrm{~cm})$.

FISHji4 and FISHji5 macro can be found on the webpage http:// paginas.fe.up.pt/ nazevedo/.

\subsection{Overlap index}

Overlap index $\Omega$ is a measure of activating regions' spatial overlap and symmetry. This value was determined using two different edges (image processing technique for finding the boundaries of cells within images detecting discontinuities in brightness): the defined by the Image method and the manual one as the ground truth [27]. After obtaining the binary images of each ROI using ImageJ, a Matlab ${ }^{\circledR}$ script (data not shown) was developed to overlap the images and subsequently to calculate the overlap index $\Omega$ according to the following equation [28]:

$\Omega=\frac{\mathrm{TP}}{\mathrm{TP}+\mathrm{FP}+\mathrm{FN}}$

where TP is the true positive area, FP is the false positive area (FP) of the ROI and FN is the false negative area or background of the ROI.

\subsection{Oligonucleotide probe design and synthesis}

Fluorescein-labeled locked nucleic acids oligonucleotides DNA/LNA and LNA/2'-OMe RNA chimeras with phosphates (PO) 


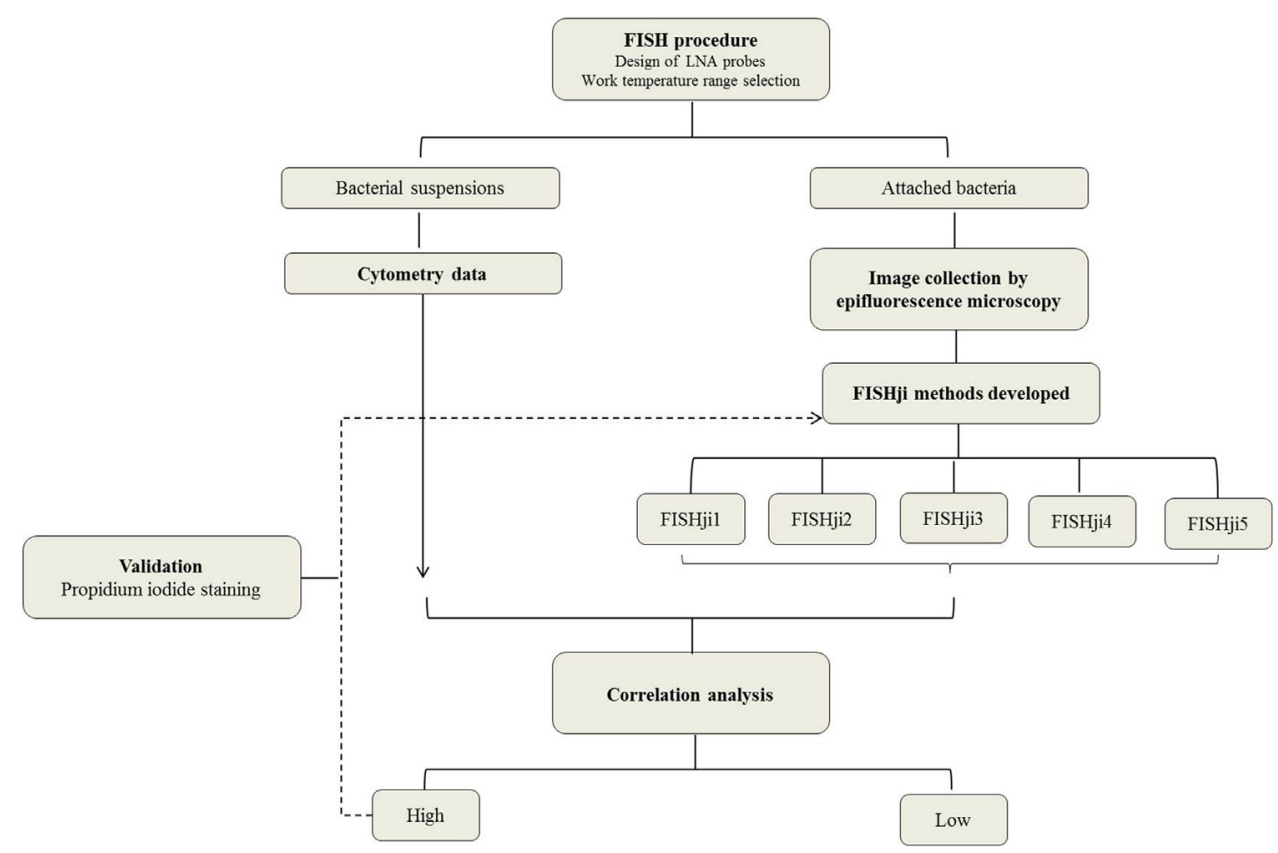

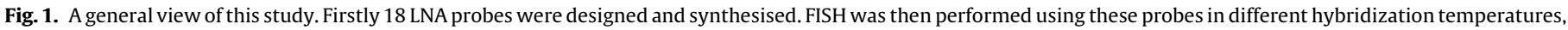

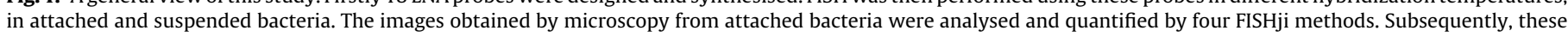

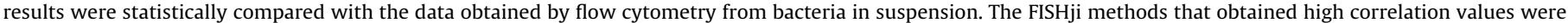
then validated using a standard staining procedure (propidium iodide).

Table 1

Probe sequences used in this study. LNA nucleotide monomers are represented with L superscript, 2'-OMe-RNA monomers in boldface letters, DNA nucleotides in capital letters, and phosphorothioate linkages by the symbol*.

\begin{tabular}{|c|c|}
\hline Probes analysed & Sequence $\left(5^{\prime}-\mathrm{FAM}-3^{\prime}\right)$ \\
\hline HP_18LNA_PO & $\mathrm{GA}^{\mathrm{L}} G A^{\mathrm{L}} C \mathrm{~T}^{\mathrm{L}} \mathrm{A} A^{\mathrm{L}} \mathrm{GC}^{\mathrm{L}} \mathrm{C} C^{\mathrm{L}} \mathrm{TC}^{\mathrm{L}} \mathrm{CT}^{\mathrm{L}} \mathrm{CC}^{\mathrm{L}}$ \\
\hline HP_18LNA_PS & $G^{*} A^{L^{*}} G^{*} A^{L^{*}} C^{*} T^{L^{*}} A^{*} A^{L^{*}} G^{*} C^{L^{*}} C^{*} C^{L *} T^{*} C^{L *} C^{*} T^{L *} C^{*} C^{L}$ \\
\hline HP_18LNA/2OMe_PO & $\mathrm{G}^{\mathrm{L}} \mathrm{AGA}{ }^{\mathrm{L}} \mathrm{CUA}{ }^{\mathrm{L}} A G \mathrm{C}^{\mathrm{L}} \mathrm{CCT}{ }^{\mathrm{L}} \mathrm{CCT}{ }^{\mathrm{L}} \mathrm{CC}$ \\
\hline HP_18LNA/2OMe_PS & $G^{L *} A^{*} G^{*} A^{L *} C^{*} U^{*} A^{L *} A^{*} G^{*} C^{L *} C^{*} C^{*} T^{L *} C^{*} C^{*} T^{L *} C^{*} C$ \\
\hline HP_15LNA_PO & $\mathrm{GA}^{\mathrm{L}} G A^{\mathrm{L}} C \mathrm{~T}^{\mathrm{L}} \mathrm{A} A^{\mathrm{L}} \mathrm{GC}^{\mathrm{L}} \mathrm{CC}^{\mathrm{L}} \mathrm{TC}^{\mathrm{L}} \mathrm{C}$ \\
\hline HP_15LNA_PO_2 & $\mathrm{GA}^{\mathrm{L}} \mathrm{GACT}^{\mathrm{L}} \mathrm{AAGC}^{\mathrm{L}} \mathrm{CCTC}^{\mathrm{L}} \mathrm{C}$ \\
\hline HP_15LNA_PS & $G^{*} A^{L^{*}} G^{*} A^{L^{*}} C^{*} T^{L^{*}} A^{*} A^{L^{*}} G^{*} C^{L^{*}} C^{*} C^{L *} T^{*} C^{L} C$ \\
\hline HP_15LNA_PS_2 & $G^{*} A^{L *} G^{*} A^{*} C^{*} T^{L *} A^{*} A^{*} G^{*} C^{L *} C^{*} C^{*} T^{*} C^{L *} C$ \\
\hline HP_15LNA/2OMe_PO & $\mathrm{G}^{\mathrm{L}} \mathrm{AGA}{ }^{\mathrm{L}} \mathrm{CUA}{ }^{\mathrm{L}} \mathrm{AG} \mathrm{C}^{\mathrm{L}} \mathrm{CCT}{ }^{\mathrm{L}} \mathrm{CC}$ \\
\hline HP_15LNA/2OMe_PS & $G^{L *} A^{*} G^{*} A^{L *} C^{*} U^{*} A^{L *} A^{*} G^{*} C^{L *} C^{*} C^{*} T^{L *} C^{*} C$ \\
\hline HP_12LNA_PO & $\mathrm{GA}^{\mathrm{L}} \mathrm{GA}^{\mathrm{L}} \mathrm{CT}^{\mathrm{L}} \mathrm{AA}^{\mathrm{L}} \mathrm{GC}^{\mathrm{L}} \mathrm{CC}^{\mathrm{L}}$ \\
\hline HP_12LNA_PS & $G^{*} A^{L^{*}} G^{*} A^{L^{*}} C^{*} T^{L^{*}} A^{*} A^{L^{*}} G^{*} C^{L^{*}} C^{*} C^{L}$ \\
\hline HP_12LNA/2OMe_PO & $\mathrm{G}^{\mathrm{L}} \mathrm{AGA}{ }^{\mathrm{L}} \mathrm{CUA}{ }^{\mathrm{L}} \mathrm{AG} \mathrm{C}^{\mathrm{L}} \mathrm{CC}$ \\
\hline HP_12LNA/2OMe_PS & $G^{*} A^{L^{*}} G^{*} A^{L^{*}} C^{*} T^{L^{*}} A^{*} A^{L^{*}} G^{*} C^{L^{*}} C^{*} C^{L}$ \\
\hline HP_10LNA_PO & $\mathrm{GA}^{\mathrm{L}} \mathrm{GA}^{\mathrm{L}} \mathrm{CT}^{\mathrm{L}} \mathrm{A} \mathrm{A}^{\mathrm{L}} \mathrm{GC}^{\mathrm{L}}$ \\
\hline HP_10LNA_PS & $G^{*} A^{L^{*}} G^{*} A^{L^{*}} C^{*} T^{L^{*}} A^{*} A^{L^{*}} G^{*} C^{L}$ \\
\hline HP_10LNA/2OMe_PO & $G^{\mathrm{L}} A G A^{\mathrm{L}} \mathrm{CUA}^{\mathrm{L}} \mathrm{AG} \mathrm{C}^{\mathrm{L}}$ \\
\hline HP_10LNA/2OMe_PS & $G^{L *} A^{*} G^{*} A^{L *} C^{*} U^{*} A^{L *} A^{*} G^{*} C^{L}$ \\
\hline
\end{tabular}

or phosphorothioates (PS) backbones (Table 1) were designed based on previous reports [29,30]. Oligonucleotides were prepared as described previously [31]. LNA and 2'OMe monomers were purchased from Exiqon (Copenhagen, Denmark) and Ribotask (Langeskov, Denmark), respectively.

\subsection{Bacterial strains and culture conditions}

H. pylori strain 26695 obtained from the American Type Culture Collection (ATCC 700392, VA USA) was maintained on trypticase soy agar (TSA) supplemented with $5 \%(\mathrm{v} / \mathrm{v})$ sheep blood (Becton Dickinson $\mathrm{GmbH}$, Germany). Single colonies were streaked onto fresh media every $48 \mathrm{~h}$ and the plates were incubated at $37^{\circ} \mathrm{C}$ under microaerobic conditions. Bacterial density was determined by the dilution of initial culture in water or saline and the absorbance was measured at $600 \mathrm{~nm}$. All experiments were performed using bac- teria in the same growth phase to avoid differences associated to cell permeabilization [32].

\subsection{Hybridization conditions on slides and in suspension by FISH}

To validate the FISHji methods, hybridization was assessed by fluorescent microscopy on slides in attached bacteria and by flow cytometry in bacterial suspensions. All probes from Table 1 were tested at temperatures between $37^{\circ} \mathrm{C}$ and $65^{\circ} \mathrm{C}$ and the fluorescence signal was quantified by both cytometry and FISHji methods. For both methodologies triplicate samples were used. In FISHji measurements we have also analysed nine images for each sample in order to study the entire sample. FISH in attached and suspended bacteria was performed as described in a previous study [31]. The cells were left at $4{ }^{\circ} \mathrm{C}$ until being analysed by cytometry. Probes were tested simultaneously at the same temperature in both samples types (attached and suspension bacteria), to minimize experimental variations between both methods (cytometry and FISHij).

\subsection{Propidium iodide (PI)}

Increasing concentrations of bacteria suspension $(500 \mu \mathrm{L})$ were mixed with propidium iodide (PI) (Invitrogen, Eugene, USA) at a concentration of $50 \mathrm{ug} / \mathrm{mL}$. For each concentration four samples were stained. Bacteria were incubated with PI for $10 \mathrm{~min}$ at room temperature. Afterwards, the samples were centrifuged during $5 \mathrm{~min}$ at $14,000 \mathrm{rpm}$ and the supernatant was discarded. Then, two of the pellets were resuspended in water and analysed by microscopy and the other two were resuspended in saline and analysed by flow cytometry.

\subsection{Microscope evaluation and quantitative analysis of fluorescence intensity}

The slides with attached bacteria were evaluated on a Leica DM LB2 epifluorescent microscope. For image acquisition, a Leica 


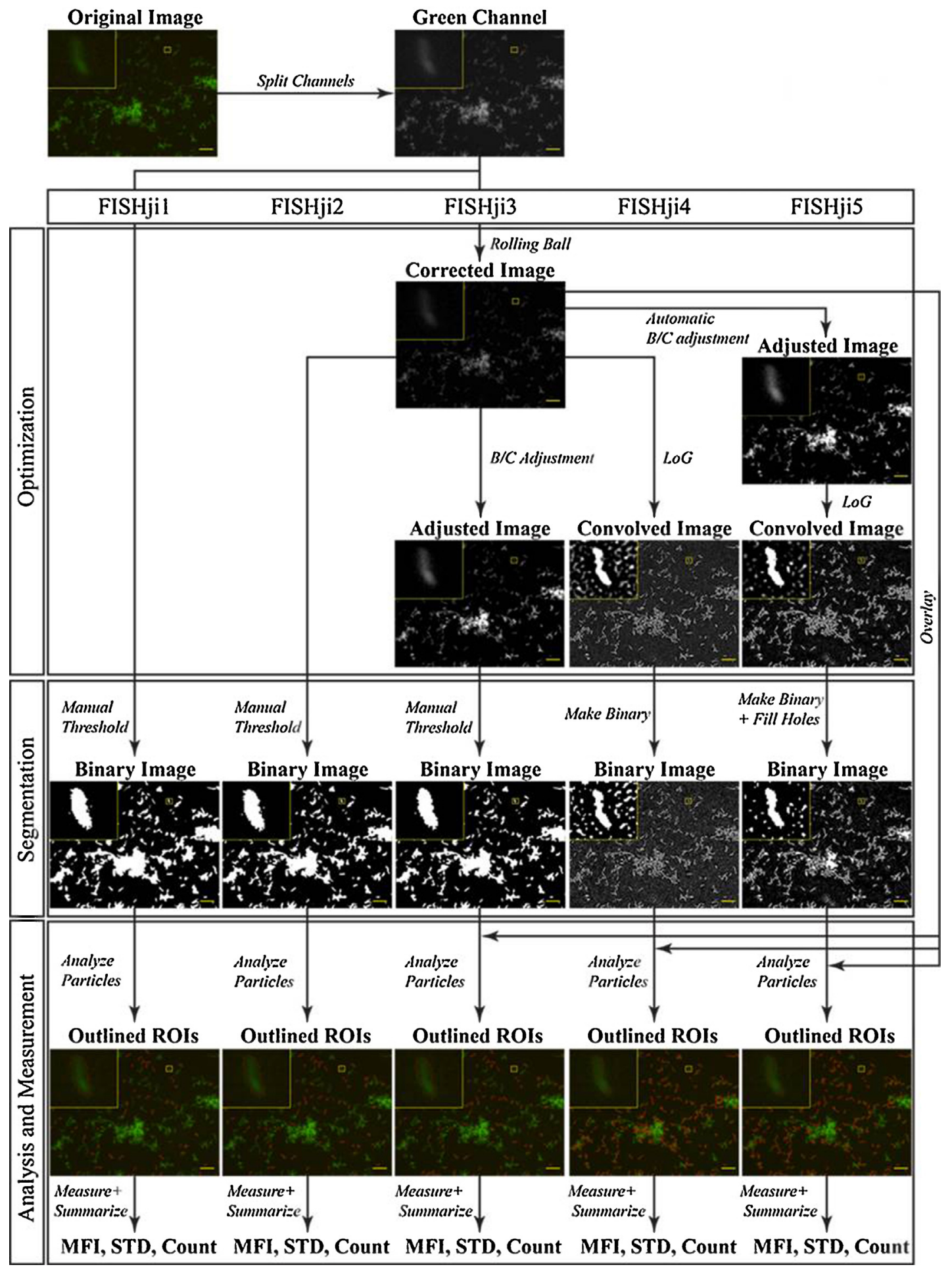

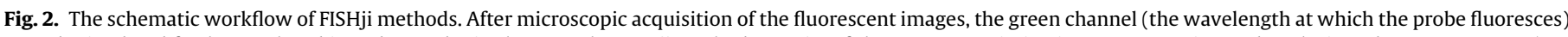

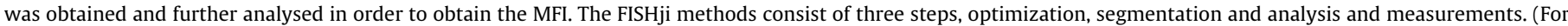
interpretation of the references to colour in this figure legend, the reader is referred to the web version of this article.)

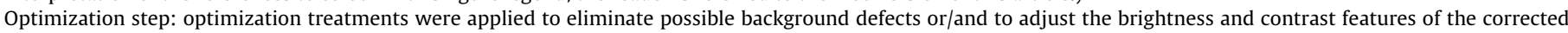

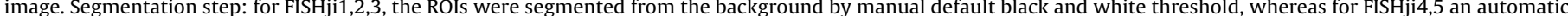

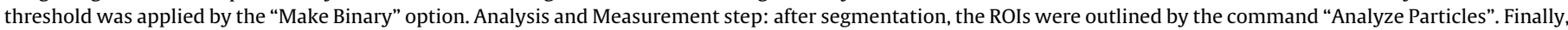

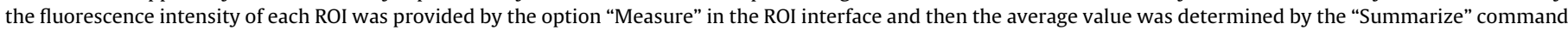

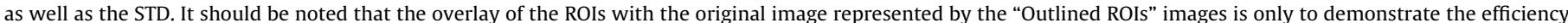

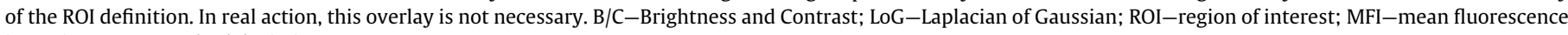
intensity; STD-standard deviation 
DFC300 FX camera (Leica Microsystems GmbHy, Germany) was used equipped with a filter capable of detect the FAM- labeling LNA probes (BP 450-490, FT 510, LP 515). The exposure time, gain and saturation values were fixed for all preparations. For image capture, Leica IM50 Image Manager, Image Processing and Archiving software was used. All experiments were performed in triplicate.

\subsection{Flow cytometry and data analysis}

The analysis of fluorescence intensities obtained by FISH in suspension was performed by flow cytometry. Flow cytometry analysis was performed using a Beckman Coulter Epics XL (Brea, USA) using the EXPO32ADC software (Beckman Coulter, Brea, USA) equipped with a low-power air-cooled $15 \mathrm{~mW}$ blue $(488 \mathrm{~nm})$ argon laser. For each sample, 20,000 events were collected. All the experiments were performed in triplicate and negative controls (samples without probes) were included for each temperature in every analysis. Flow cytometric analyses of samples were performed based on forward scatter, side scatter and FL-1. Fluorescence was detected on the FL1 channel (BP530/30). Amplification was carried out using logarithmical scales.

\subsection{Statistical and correlation analysis}

Results are compared using one-way analysis of variance (ANOVA) by applying Tukey multiple-comparisons test, using GraphPad Prism version 5 software (GraphPad Sofware, San Diego, USA). All tests were performed with a confidence level of $95 \%$.

\section{Results and discussion}

\subsection{Comparison between FISHji measurements and quantification by cytometry}

In this paper we developed and validated five semi-automatic or automatic fluorescent intensity quantification methods, called FISHji, capable of quantifying the MFI. The settings and characteristics of each FISHji method developed are described in Table 2. As ground truth, the MFI of the hybridization experiments was also quantified using flow cytometry (gold standard method in fluorescence quantification) at the same temperature as the hybridizations performed in attached bacteria.

In order to assess which FISHji method provide MFI values closer to the ones obtained by cytometry, the correlation between results from each FISHji (semi-automatic and automatic methods) and cytometry was calculated (Table 3 ). The three semi-automatic methods (FISHji 1, FISHji 2 and FISHji 3) present different results when compared to the cytometry. FISHji1 showed the lowest correlation with flow cytometry (Table 3). FISHji 2 showed a better correlation than FISHji 1, which suggests that, the performance of the optimization steps improved MFI quantification efficiency. The FISHji3 revealed to be the best semi-automatic method demonstrating that the application of a B/C adjustment after subtraction of the background contributed to a more effective MFI quantification.

Nevertheless, semi-automatic methods showed to be very timeconsuming when a large number of samples have to be processed (Table 1), and since a routine protocol to perform fluorescence quantification should be fast and easy to work with, the need for an automatic method becomes clear. The automatic threshold definition is much more difficult to perform since one image is never equal to the other. Hence, has emerged the need for a more suitable and automatic segmentation technique. Previous studies have used LoG convolution to automatically enhance ROIs from the background with good outcomes [33]. Herein, FISHji4 was designed in which this approach was used to do both smoothing and edge detection. A high correlation between our plugin results and flow cytometry analysis for the automatic FISHji4 was observed (Table 3). However the correlation factor is lower comparatively to the semi-automatic FISHji3. This result might have been influenced by cells from aggregates that were selected and subsequently counted due to the elevated sensibility of the convolution (Fig. 2). Further, artefacts are not possible to discard in the ROI manager.

In an attempt to increase the automatic performance, a fifth method was designed (FISHji5) specifically to blurred regions after subtracted background, thereby connecting nearby cells (i.e. belonging to aggregates) and subsequently impairing the aforementioned problem. A high correlation between FISHji 5 and flow cytometry was also observed (Table 3). Contrary to expected, no significant differences emerged between both automatic methods. Visual image analysis allowed to conclude that the influence of aggregates in the present study was overestimated and auto $\mathrm{B} / \mathrm{C}$ adjustment in ROIs with high fluorescence intensity increased the area of analysis, selecting false positive regions.

The results obtained for the correlation coefficient for FISHji 3, 4 and 5 are shown in detail in Fig. 3. where each data point consists of two parameters: the X-axis values corresponds to the mean of fluorescence intensity obtained by cytometry with a specific probe and temperature and the $\mathrm{Y}$-axis values represent the mean value of fluorescence intensity obtained by FISHji in the same conditions. The average Pearson product moment correlation coefficient from all our biological repeats show a high level of correlation between the FISHji results and cytometry analysis, $r=0.819(0.733-0.880)$ in FISHji3.

One of the main factors that is influencing the results is the implementation of optimization steps, that reflects on the efficiency of the definition of the edges of the ROI. In order to verify this influence in the MFI quantification, we calculated the overlap index $\Omega$, as a measurement of the overlap performance between the edges defined by the implemented methods and the ground truth. This analysis is a commonly used method in several processing image studies, however it can suffer different adaptations depending on the purpose of the analysis [34,35]. FISHj1 showed the lowest mean $\Omega$, indicating that the application of optimization steps before image segmentation improved the definition of the cell. In general, the overlapping results were congruent with the correlation factors for each method, indicating that the cell definition contributes largely to the MFI quantification.

To evaluate whether the choice of the optimization steps and the consequent image analised had influence in the MFI quantification, FISH images were reanalysed by the FISHji4 and FISHji5 methods, however now redirecting the binary mask to the original image instead of the corrected one. The low correlation coefficients obtained $(r=0.317$ and $r=0.231$, for FISHji4 and FISHji5) respectively showed that the use of corrected images (after optimization steps) is essential in these analysis. Additionally, the original images can contain many different artefacts and autofluorescence signals that may blur or omit the real fluorescence value of the cell. The application of the "rolling ball" algorithm to the original green channel can correct background heterogeneity and eliminate some artefacts, contributing to a more realistic MFI quantification when compared to the cytometry. Therefore, the choice of the analysed image showed to be a major point in the MFI quantification, whereas the application of optimization steps before thresholding takes a relevant part in the efficiency of the definition of the cell, contributing in a smaller scale to the MFI quantification performance.

We also evaluated the influence of hybridization temperature in FISHji methods. Although there were differences between different hybridization temperatures, overall, the correlation coefficients were higher than those in the global analysis. As an example, we showed in Fig. 4 the results for hybridization performed at $45^{\circ} \mathrm{C}$ and $55^{\circ} \mathrm{C}$ for FISHji3. In both cases we observed a high correla- 
Table 2

Comparisons between the five FISHji methods. The different methods were compared relatively to these general features.

\begin{tabular}{|c|c|c|c|c|c|}
\hline General features & FISHji1 & FISHji 2 & FISHji3 & FISHji4 & FISHji5 \\
\hline Operational mode & Semi-automatic & Semi-automatic & Semi-automatic & Automatic & Automatic \\
\hline Image for analysis & Single image & Single image & Single image & Multiple images & Multiple images \\
\hline Analysis speed per image & $\sim 50 \mathrm{~s}$ & $\sim 50 \mathrm{~s}$ & $\sim 1 \mathrm{~min} 20 \mathrm{~s}$ & $4 \mathrm{~s}$ & $4 \mathrm{~s}$ \\
\hline Cell segmentation & Manual threshold & Manual threshold & Manual threshold & Automatic threshold & - \\
\hline Rolling Ball & - & + & + & + & + \\
\hline Manual B/C & - & - & + & + & - \\
\hline $\mathrm{B} / \mathrm{C}$ auto & - & - & - & - & + \\
\hline LoG & - & - & - & + & + \\
\hline Fill Holes & - & - & - & - & + \\
\hline Analysed image & Original & Corrected & Corrected & Corrected & Corrected \\
\hline
\end{tabular}

Table 3

Comparison between each FISHji method values and flow cytometry data.

\begin{tabular}{|c|c|c|c|c|c|}
\hline Measurements & FISHji 1 & FISHji 2 & FISHji 3 & FISHji 4 & FISHji 5 \\
\hline$\Omega$ & 0.46 & 0.51 & 0.54 & 0.63 & 0.62 \\
\hline Correlation coefficient ( $\mathrm{p}$-value) & $0.456(<0.0001)$ & $0.751(<0.0001)$ & $0.819(<0.0001)$ & $0.723(<0.0001)$ & $0.717(<0.0001)$ \\
\hline
\end{tabular}

* Flow cytometry is used as a ground truth for calculating the correlation coefficient and the p-value.

A

FISHji3

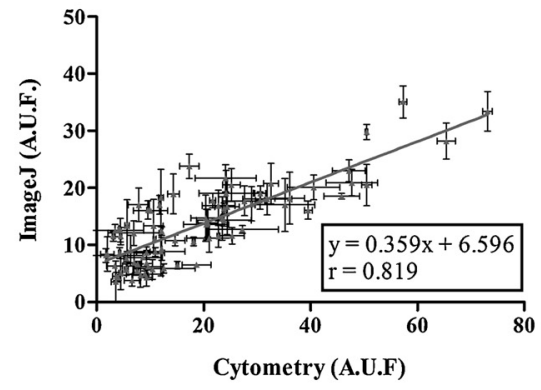

$\mathbf{B}$

FISHji4

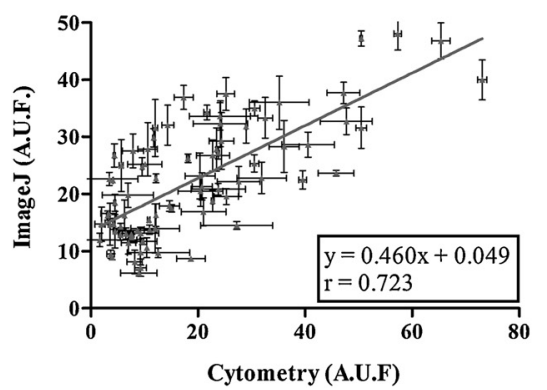

C

FISHji5

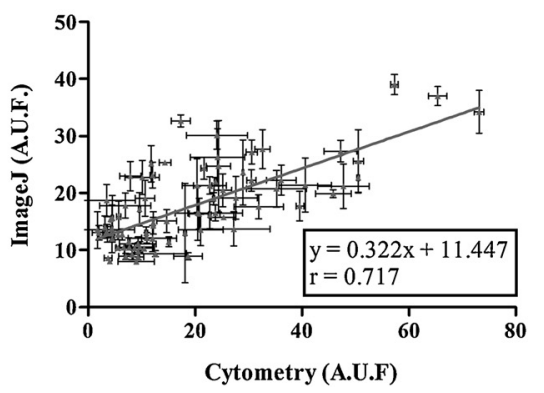

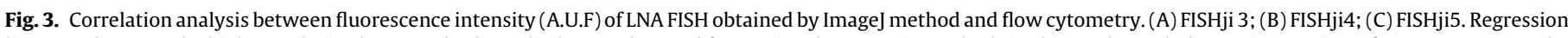

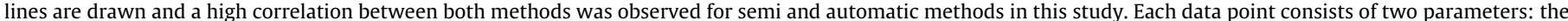

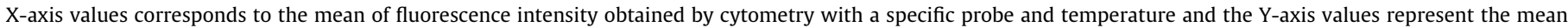
value of fluorescence intensity obtained by FISHji in the same conditions.

A

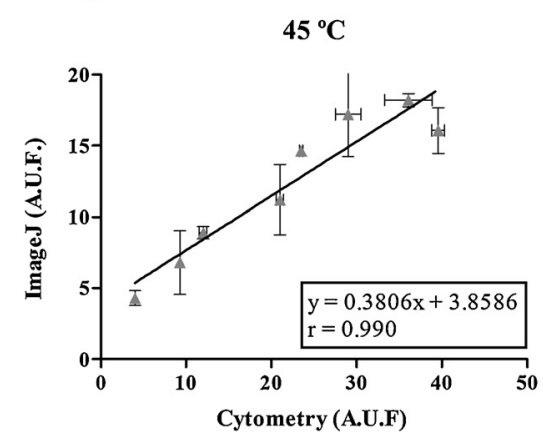

B

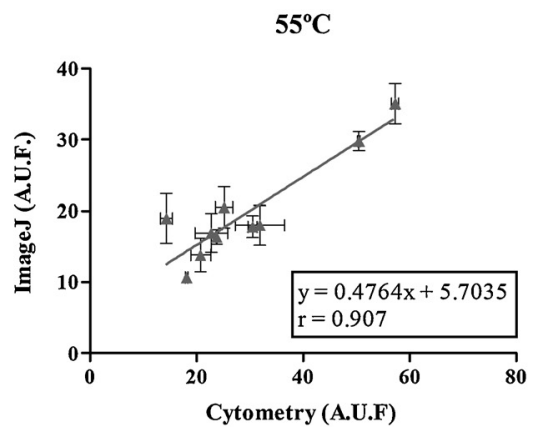

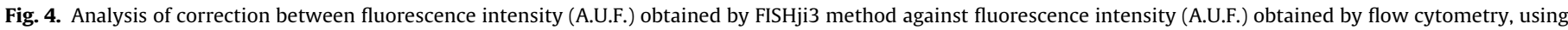
hybridization temperatures. (A) $45^{\circ} \mathrm{C}$; (B) $55^{\circ} \mathrm{C}$. Regression lines are drawn and the $\mathrm{r}$ is displayed for each data comparison. 


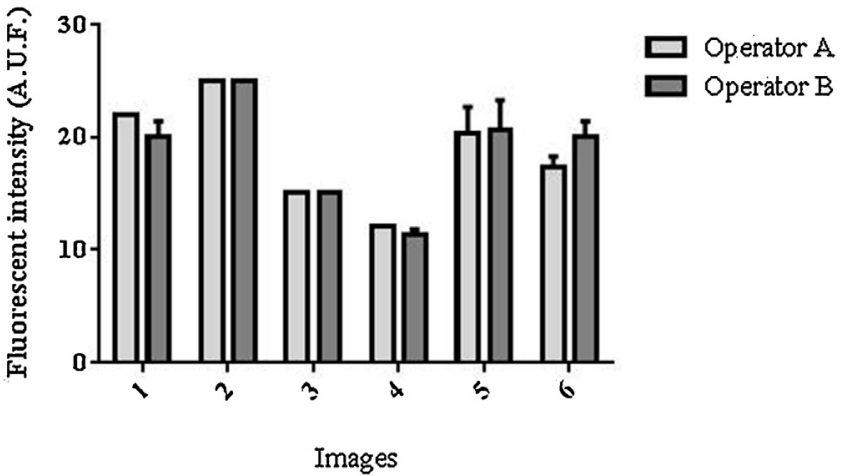

Fig. 5. Variability test between operators in FISHji 3.Fluorescence intensity was quantified for 6 images from 6 different experiments. Each image was independently analysed by each operator. Differences were observed between operators A and B were statistically non-significant ( $p \geq 0.05$ ). A.U.F.: Arbitrary units of fluorescence.

tion $\mathrm{r}=0.946(0.724-0.990)$ for $45^{\circ} \mathrm{C}$ and $\mathrm{r}=0.907(0.647-0.978)$ for $55^{\circ} \mathrm{C}$. This suggests that the lower correlation values obtained for the FISHji3 using the entire set of data might also be due to some experimental variation.

Because FISHji3 is a semi-automatic method, it requires manual definition of a threshold by the operator. In order to evaluate the influence of different operators in MFI quantification, two different operators have quantified the MFI of the same images. Our results (Fig. 5) did not show statistically significance differences between the MFI values determined by each operator $(p>0.05)$. The same results were obtained for FISHji1 and FISHji2 (see Supplementary material Fig. S1 in the online version at DOI: 10.1016/j.bej.2016.04. 001).

While FISHji3 shows higher correlation with cytometer and is operator-independent for threshold, automatic methods FISHji4 or FISHji5 could be the key to development of a routine analysis for multiple FISH images. In this sense, automatic quantification without the need of expensive equipment (such as cytometer) would be recommended. Comparisons between FISHji3, FISHji4 and FISHji5 showed high levels of correlation in both cases (Fig. 6), suggestion that both FISHji4 and FISHji5 offer the ability to automatically analyse considerable amounts of image data.

In the present study, the main cornerstone of fluorescence intensity quantification was cell segmentation (i.e. definition of the ROIs). Indeed, segmentation can be very difficult due to the complexity of original images, represented by combination of different features like (1) autofluorescence; (2) background heterogeneity; (3) presence of non-cell objects and aggregates (herein considered as artefacts). Therefore, a very important issue to take in consideration when using this tool is the high dependence on image quality [3]. There are several factors which may interfere with the quality of the images, such as the numeral aperture (NA) of the objective, filed illumination of the lens, the refractive index of the embedding medium, use of coverslip, stability of the light source, the ratio between emission and excitation wavelength, amongst others [36]. Therefore, we always used the same settings in the microscope for every experiment to minimize these factors. Although all parameters associated with the image collection were kept constant, such as exposures times, illumination and focus, it was impossible to guarantee that the fluorescent lamp would produce the same light intensity during the whole of the experiments.

\subsection{Correlation between FISHji measurements and quantification by cytometry using PI staining}

In order to address the use of FISHji methods in measuring fluorescence obtained by means other than FISH, we performed the staining of $H$. pylori samples with PI. Therefore, we analysed the same sample by microscopy (and consequently the fluorescence intensity by FISHji 3, 4 and 5 methods) and cytometry. All
$\mathbf{A}$

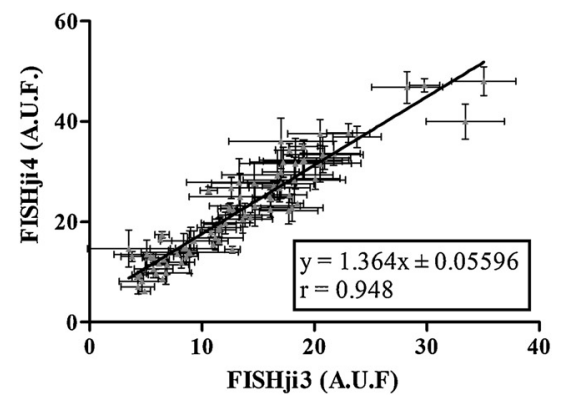

B

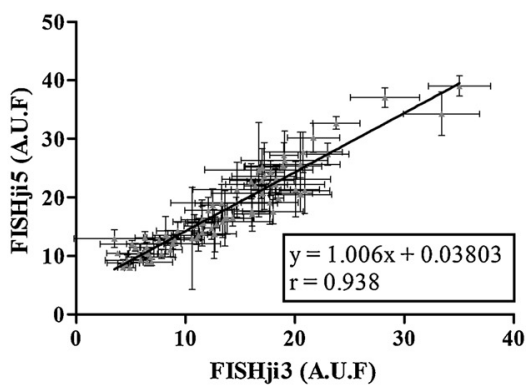

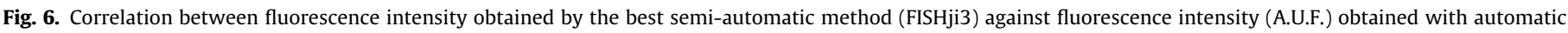
methods FISHji4 (A) and FISHji5 (B). A.U.F.: Arbitrary units of fluorescence.

A

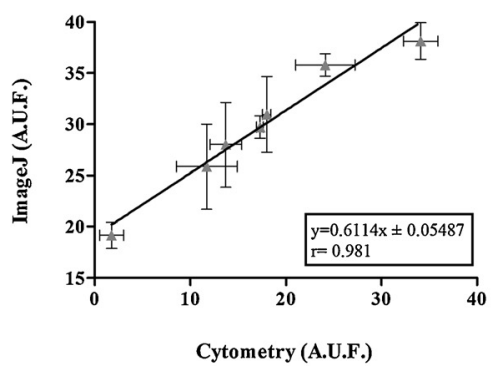

B

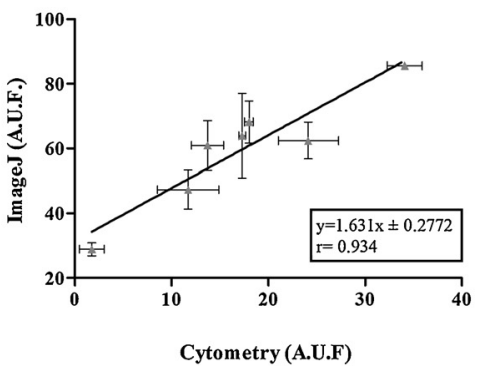

C

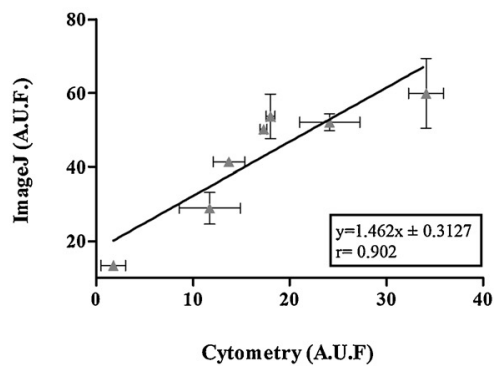

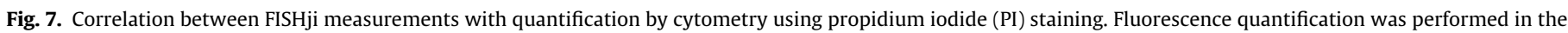
red channel. (A) FISHji3; (B) FISHji4; (C) FISHji5. A.U.F.: Arbitrary units of fluorescence. 
the parameters were maintained in relation to the previous study. Our results (Fig. 7) showed that there is a high correlation with the FISHji3 method $r=0.981$ (0.8690-0.9972), with high significance $(\mathrm{p}<0.0001)$. The analysis with the automatic methods also showed high correlation $r=0.934(0.6137-0.9906)$ and $r=0.902$ (0.4650-0.9856) for FISHji4 and FISHji5, respectively.

These results prove that FISHji could be used for any type of fluorescence procedures and that the correlation coefficients can be even higher for more reproducible experimental procedures. For that reason, we can conclude that a higher correlation relatively to the FISH experiments was probably due to several factors which interfere with signal fluorescence in this type of technique. It is already know that fluorescence is a complex phenomenon controlled by many external factors [37], and that small variations may lead to a significant change in the signal intensity.

\section{Conclusion}

In biological sciences, fluorescence-based methods are crucial to study, analyse or identify microorganisms. Here, we present FISHji methods to quantify fluorescence in images from biological samples obtained by microscopy. We show that the semi-automatic FISHji3 and automatic FISHji4 and FISHji5 have high accuracy in different settings such as FISH and PI staining. Because FISHji4 presents better results comparatively to FISHji5, we consider that this method should be preferred for fluorescence quantifications. Nonetheless, it is important to bear in mind that variations in the types of samples (e.g. cell size or aggregation), might imply that for specific situations, FISHji5 might be more adequate.

Since automated methods are able to simultaneously analyse multiple images and are consequently faster, we provide an easy and freely available access not only to FISHji4 but also to FISHji5 for non-commercial use at http://paginas.fe.up.pt/ nazevedo/.

\section{Author contributions}

Conceived and designed the experiments: SF, DC, NFA. Performed the experiments: SF, DC. Analyzed the data: SF, DC, AL, NG, NFA. Tool development: SF, DC, AL; Contributed reagents/materials/analysis tools: $\mathrm{SF}, \mathrm{DC}, \mathrm{PM}, \mathrm{CF}, \mathrm{NFA}$. Wrote the paper: SF, DC, AL, NG, PM, CF, NFA.

\section{Acknowledgments}

This work was financially supported by: Project UID/EQU/00511/2013-LEPABE, by the FCT/MEC with national funds and when applicable co-funded by FEDER in the scope of the P2020 Partnership Agreement; Project NORTE-07-0124-FEDER-000025-RL2_Environment\&Health,

by FEDER funds through Programa Operacional Factores de Competitividade-COMPETE, by the Programa Operacional do Norte (ON2) program and by national funds through FCT-Fundaça o para a Ciência e a Tecnologia; DNA mimics Research Project PIC/IC/82815/2007, PhD Fellowship SFRH/BD/72999/2010 and Post-Doctoral fellowship SFRH/BPD/78846/2011.

\section{References}

[1] V. Wiesmann, D. Franz, C. Held, C. Munzenmayer, R. Palmisano, T. Wittenberg, Review of free software tools for image analysis of fluorescence cell micrographs, J. Microsc. 257 (1) (2015) 39-53.

[2] T.J. Collins, Image for microscopy, Biotechniques 43 (Suppl. 1) (2007) 25-30.

[3] S.M. Hartig, Basic image analysis and manipulation in ImageJ, Curr. Protoc. Mol. Biol. (2013), http://dx.doi.org/10.1002/0471142727.mb1415s102, Chapter 14: p. Unit14 15.
[4] A. Shivanandan, A. Radenovic, I.F. Sbalzarini, MosaicIA: an ImageJ/Fiji plugin for spatial pattern and interaction analysis, BMC Bioinf. 14 (2013) 349

[5] P.W.Winter, H. Shroff, Faster fluorescence microscopy: advances in high speed biological imaging, Curr. Opin. Chem. Biol. 20 (0) (2014) 46-53.

[6] F.P. Cordelières, S. Bolte, Chapter 21-experimenters' guide to colocalization studies: finding a way through indicators and quantifiers, in practice, in: C.W. Jennifer, W. Torsten (Eds.), Methods in Cell Biology, Academic Press, 2014, pp. 395-408.

[7] P.W. Winter, H. Shroff, Faster fluorescence microscopy: advances in high speed biological imaging, Curr. Opin. Chem. Biol. 20 (2014) 46-53.

[8] J.F. Dorn, G. Danuser, G. Yang, Computational processing and analysis of dynamic fluorescence image data, in: F.S. Kevin (Ed.), Methods in Cell Biology, Academic Press, 2008, pp. 497-538.

[9] A.R. Cohen, Extracting meaning from biological imaging data, Mol. Biol. Cell 25 (22) (2014) 3470-3473

[10] S. van Teeffelen, J.W. Shaevitz, Z. Gitai, Image analysis in fluorescence microscopy: bacterial dynamics as a case study, Bioessays 34 (5) (2012) 427-436.

[11] R.I. Amann, L. Krumholz, D.A. Stahl, Fluorescent-oligonucleotide probing of whole cells for determinative, phylogenetic, and environmental studies in microbiology, J. Bacteriol. 172 (2) (1990) 762-770.

[12] E.F. DeLong, G.S. Wickham, N.R. Pace, Phylogenetic stains: ribosomal RNA-based probes for the identification of single cells, Science 243 (4896) (1989) 1360-1363.

[13] R.I. Amann, W. Ludwig, K.H. Schleifer, Phylogenetic identification and in situ detection of individual microbial cells without cultivation, Microbiol. Rev. 59 (1) (1995) 143-169.

[14] K. Kubota, A. Ohashi, H. Imachi, H. Harada, Improved in situ hybridization efficiency with locked-nucleic-acid-incorporated DNA probes, Appl. Environ. Microbiol. 72 (8) (2006) 5311-5317.

[15] L. Cerqueira, R.M. Fernandes, R.M. Ferreira, F. Carneiro, M. Dinis-Ribeiro, C. Figueiredo, C.W. Keevil, N.F. Azevedo, M.J. Vieira, PNA-FISH as a new diagnostic method for the determination of clarithromycin resistance of Helicobacter pylori, BMC Microbiol. 11 (2011) 101

[16] T. Yamamoto, M. Nakatani, K. Narukawa, S. Obika, Antisense drug discovery and development, Future Med. Chem. 3 (3) (2011) 339-365.

[17] A.N. Elayadi, D.A. Braasch, D.R. Corey, Implications of high-affinity hybridization by locked nucleic acid oligomers for inhibition of human telomerase, Biochemistry 41 (31) (2002) 9973-9981.

[18] N.M. Bell, J. Micklefield, Chemical modification of oligonucleotides for therapeutic, bioanalytical and other applications, ChemBioChem 10 (17) (2009) 2691-2703.

[19] J. Konsti, J. Lundin, M. Jumppanen, M. Lundin, A. Viitanen, J. Isola, A public-domain image processing tool for automated quantification of fluorescence in situ hybridisation signals, J. Clin. Pathol. 61 (3) (2008) $278-282$.

[20] H. Daims, S. Lucker, M. Wagner, daime, a novel image analysis program for microbial ecology and biofilm research, Environ. Microbiol. 8 (2) (2006) 200-213.

[21] K. Stoecker, C. Dorninger, H. Daims, M. Wagner, Double labeling of oligonucleotide probes for fluorescence in situ hybridization (DOPE-FISH) improves signal intensity and increases rRNA accessibility, Appl. Environ. Microbiol. 76 (3) (2010) 922-926.

[22] R. Hatzenpichler, E.V. Lebedeva, E. Spieck, K. Stoecker, A. Richter, H. Daims, M. Wagner, A moderately thermophilic ammonia-oxidizing crenarchaeote from a hot spring, Proc. Natl. Acad. Sci. U. S. A. 105 (6) (2008) 2134-2139.

[23] S.D. Weber, W. Ludwig, K.H. Schleifer, J. Fried, Microbial composition and structure of aerobic granular sewage biofilms, Appl. Environ. Microbiol. 73 (19) (2007) 6233-6240.

[24] C.A. Schneider, W.S. Rasband, K.W. Eliceiri, NIH Image to Image]: 25 years of image analysis, Nat. Methods 9 (7) (2012) 671-675.

[25] S.R. Sternberg, Biomedical image processing, Computer 16 (1) (1983) 22-34.

[26] J.S. Chen, A. Huertas, G. Medioni, Fast convolution with Laplacian-of-Gaussian masks, IEEE Trans. Pattern Anal. Mach. Intell. 9 (4) (1987) 584-590.

[27] L. Hogeweg, C.I. Sánchez, P.A. de Jong, P. Maduskar, B. van Ginneken, Clavicle segmentation in chest radiographs, Med. Image Anal. 16 (8) (2012) 1490-1502.

[28] G. Gerig, M. Jomier, M. Chakos, Valmet: a new validation tool for assessing and improving 3D object segmentation, in: W. Niessen, M. Viergever (Eds.), Medical Image Computing and Computer-Assisted Intervention-MICCAI, Springer Berlin Heidelberg, 2001, pp. 516-523.

[29] R. Kumar, S.K. Singh, A.A. Koshkin, V.K. Rajwanshi, M. Meldgaard, J. Wengel, The first analogues of LNA (locked nucleic acids): phosphorothioate-LNA and 2'-thio-LNA, Bioorg. Med. Chem. Lett. 8 (16) (1998) 2219-2222.

[30] Y. You, B.G. Moreira, M.A. Behlke, R. Owczarzy, Design of LNA probes that improve mismatch discrimination, Nucleic Acids Res. 34 (8) (2006) pe60.

[31] S. Fontenete, N. Guimaraes, M. Leite, C. Figueiredo, J. Wengel, N. Filipe Azevedo, Hybridization-based detection of Helicobacter pylori at human body temperature using advanced locked nucleic acid (LNA) probes, PLoS One 8 (11) (2013) e81230.

[32] B. Bottari, E.D. Gatti, M. Neviani, Fish, in: T. Liehr (Ed.), Food Microbiology, Springer-Verlag, Berling, 2009

[33] M. Haidekker, Advanced Biomedical Image Analysis, Wiley, 2010, pp. 528.

[34] H. Zaidi, M. Abdoli, C.L. Fuentes, I.M. El Naqa, Comparative methods for PET image segmentation in pharyngolaryngeal squamous cell carcinoma, Eur. J. Nucl. Med. Mol. Imaging 39 (5) (2012) 881-891. 
[35] K.H. Zou, S.K. Warfield, A. Bharatha, C.M. Tempany, M.R. Kaus, S.J. Haker, W.M. Wells, 3rd, F.A. Jolesz, R. Kikinis, Statistical validation of image segmentation quality based on a spatial overlap index, Acad. Radiol. 11 (2) (2004) 178-189.

[36] G. Kumar, R.M. Zucker, Fluorescence in situ hybridization (FISH) imaging, in: Clive R. Taylor, Lars Rudbeck (Eds.), Immunohistochemical Staining Methods Education Guide, Sixth Ed., Dako, Denmark, 2013.
[37] H.J. Tanke, Fluorescence microscopy for quantitative fluorescence in situ hybridization analysis, in: P.D.M. Andreeff (Ed.), Introduction to Fluorescence In Situ Hybridization: Principles and Clinical Applications, Wiley-Liss, 1999. 